\title{
Uniform boundedness and pattern formation for Keller-Segel systems with two competing species
}

\author{
Yuanyuan Zhang , Ce Huang ${ }^{\dagger}$ and Li Xia ${ }^{\ddagger}$
}

\begin{abstract}
We consider a fully parabolic reaction-advection-diffusion system over two-dimensional bounded domain endowed with the homogeneous Neumann boundary conditions. This system models the chemotactic movements and population dynamics of two Lotka-Volterra competing microbial species attracted by the same chemical stimulus. We obtain the global existence of classical solutions to this two-dimensional system and prove that the global solutions are uniformly bounded in their $L^{\infty}$-norms. Our result does not require chemotaxis rates to be small or decay rate to be large. Moreover numerical simulations are performed to illustrate the formation and qualitative properties of stable orand time-periodic spatially-inhomogeneous patterns of the system. Our theoretical and numerical findings illustrate that this two-dimensional chemotaxis model is able to demonstrate very interesting and complicated spatial-temporal dynamics.

Keywords: global existence, uniform boundedness, two species chemotaxis model, pattern formation.
\end{abstract}

\section{Introduction}

In this paper, we study the global existence and uniform boundedness of classical solutions to the following reaction-diffusion system

$$
\begin{cases}u_{t}=\nabla \cdot\left(d_{1} \nabla u-\chi_{1} u \nabla w\right)+\mu_{1}\left(1-u-a_{1} v\right) u, & x \in \Omega, t>0, \\ v_{t}=\nabla \cdot\left(d_{2} \nabla v-\chi_{2} v \nabla w\right)+\mu_{2}\left(1-a_{2} u-v\right) v, & x \in \Omega, t>0, \\ \tau w_{t}=\Delta w-\lambda w+u+v, & x \in \Omega, t>0, \\ \frac{\partial u}{\partial \nu}=\frac{\partial v}{\partial \nu}=\frac{\partial w}{\partial \nu}=0, & x \in \partial \Omega, t>0, \\ u(x, 0)=u_{0}(x), v(x, 0)=v_{0}(x), w(x, 0)=w_{0}(x), & x \in \Omega,\end{cases}
$$

where $\Omega$ is a bounded domain with smooth boundary $\partial \Omega ; \nabla$ and $\Delta$ are the gradient operator and Laplace operator respectively; $a_{i}, d_{i}, \mu_{i}, \chi_{i}, i=1,2$, and $\lambda, \tau$ are assumed to be constants.

System (1.1) was proposed by J. Tello and M. Winkler in [22] to study the spatial-temporal evolution of two competing microbial species which are attracted by the same chemical stimulus (chemoattractant) secreted by both species. In (1.1), $u(x, t)$ and $v(x, t)$ represent the cellular population densities of the two competing bacterial species at space-time location $(x, t) \in \Omega \times \mathbb{R}^{+}$

\footnotetext{
${ }^{*}$ School of Securities and Futures, Southwestern University of Finance and Economics, Chengdu, Sichuan 611130, China. The work of Yuanyuan Zhang is supported by Department of Education, Suchuan.

${ }^{\dagger}$ School of Public Finance and Taxation, Southwestern University of Finance and Economics, Chengdu, Sichuan 611130, China. The work of Ce Huang is supported by NSF of China

${ }^{\ddagger}$ School of Mathematics and Statistics, Guangdong University of Finance and Economics, Guangzhou, Guangdong 510320, China. LX is partially supported by NSF of China (11201311). Correspondence to xaleysherry@163.com, .
} 
respectively, and $w(x, t)$ is the concentration of the chemical. It is assumed in [22] that $\chi_{1}, \chi_{2}$ and $\lambda$ are positive constants, which interprets the biological phenomenon that both bacteria direct their movements chemotactically along the gradient of chemoattractant concentration, while the chemical is produced by both species and is consumed by certain enzyme meanwhile. The kinetics of the species are assumed to be of the classical Lotka-Volterra type and $a_{1}, a_{2}$ interpret the levels of inter-specific competition. The results in [22] can be summarized as follows.

Theorem 1.1. (Tello, Winkler [22]) Let $\Omega \subset \mathbb{R}^{N}, N \geq 1$, be a bounded domain with smooth boundary $\partial \Omega$. Let $\tau=0, d_{1}=d_{2}=1$. Assume that $0 \leq a_{1}, a_{2}<1$ and the following hypothesis is satisfied

$$
2(\chi+\xi)+a_{1} \mu_{2}<\mu_{1}, 2(\chi+\xi)+a_{2} \mu_{1}<\mu_{2},
$$

then for any positive initial data $\left(u_{0}, v_{0}\right) \in C^{0}(\bar{\Omega}) \times C^{0}(\bar{\Omega})$, (1.1) has a unique classical solution $(u(x, t), v(x, t), w(x, t))$ which is global and bounded; moreover, the positive equilibrium $(\bar{u}, \bar{v}, \bar{w})=\left(\frac{1-a_{1}}{1-a_{1} a_{2}}, \frac{1-a_{2}}{1-a_{1} a_{2}}, \frac{2-a_{1}-a_{2}}{\lambda\left(1-a_{1} a_{2}\right)}\right)$ is a global attractor of (1.1) in the sense

$$
\|u(\cdot, t)-\bar{u}\|_{L^{\infty}}+\|v(\cdot, t)-\bar{v}\|_{L^{\infty}}+\|w(\cdot, t)-\bar{w}\|_{L^{\infty}} \rightarrow 0, t \rightarrow \infty .
$$

Theorem 1.1 indicates that the equilibrium $(\bar{u}, \bar{v}, \bar{w})$ is globally asymptotically stable when the chemotactic forces are weak compared to the intrinsic cellular growth rates, therefore the stationary system of (1.1) has no positive solutions other than $(\bar{u}, \bar{v}, \bar{w})$ under condition (1.2). From the view point of mathematical modeling, it is interesting to study the nonconstant steady states of the two species chemotaxis model (1.1).

For $\Omega=(0, L)$, Q. Wang et al [26] proved the global existence and uniform boundedness of positive solutions to (1.1). Moreover, rigorous bifurcation analysis is carried out to study the existence and stability of nonconstant positive steady states. Very recently, they proved in [27] that when $\mu_{1}=\mu_{2}=0, \Omega$ is a bounded domain in $\mathbb{R}^{2}$, under the following condition on the initial cellular populations,

$$
\frac{\chi}{d_{1}} \int_{\Omega} u_{0}+\frac{\xi}{d_{2}} \int_{\Omega} v_{0}<4 \pi
$$

the solutions to (1.1) are global and uniformly bounded in time. For $\mu_{1}=\mu_{2}=0$, blow-ups of (1.1) for $\Omega=\mathbb{R}^{2}$ have been studied by various authors in [4, 7, 8, 10]

One of the most interesting phenomena in chemotaxis is the cellular aggregation in which initially homogeneously distributed cells migrate along the gradient of concentration of certain chemical that they release, group with the nearby cell, and eventually form into a stable fruiting body within the habitat. There are two well-established methods in literature to model this phenomenon in mathematics. Time-dependent system can model cellular aggregation as $L_{\infty^{-}}$ norm of the solutions goes to infinity, therefore the cellular aggregation is simulated by $\delta$ functions. Mathematical analysis of the blow-up solutions to Keller-Segel models was initiated in $[6,17]$ formally, while local blow-up profile was rigorously constructed in [12] over a 2D domain. Another approach is to prove that positive solutions exist globally in time and converge to bounded steady states as time involves. Then we can use steady states with concentrating properties such as boundary or interior spikes to model the cell aggregation phenomenon. See $[5,19,20,23,24]$ for details in this direction. Similar approach has been taken to model the segregation phenomenon in Lotka-Volterra competition models in mathematical ecology. See $[15,16,25]$ and the references therein for works in this direction.

Apparently the concentrating property of the $\delta$-function is intrinsically connected to the phenomenon of cellular aggregation, such blow-up solution is not realistic from the viewpoint of mathematical modeling since chemical concentration can not be infinity at a spot in the domain; moreover it brings challenges to numerical simulations and rigorous analysis of the states after blow-up. We shall take the second approach in our paper and it is the first goal of the current work to investigate the global existence and uniform boundedness of classical solutions to system (1.1). Our first main result reads as follows. 
Theorem 1.2. Let $\Omega \subset \mathbb{R}^{2}$ be a bounded domain with smooth boundary $\partial \Omega$ and $\nu$ is the unit outer normal on the boundary. Assume that the constants $a_{i}, d_{i}, \mu_{i}, \chi_{i}, i=1,2, \lambda$ are positive and $\tau$ is nonnegative. Suppose that $u_{0} \in C^{\alpha}(\Omega), v_{0} \in C^{\alpha}(\Omega)$ and $w_{0} \in W^{2, p}(\Omega)$ for some $\alpha \in(0,1)$ and $p>2$ satisfying $u_{0}, v_{0}, w_{0} \geq 0, \not \equiv 0$ in $\Omega$. Then there exists a unique global classical solution $(u, v, w) \in C^{0}(\bar{\Omega} \times[0, \infty)) \cap C^{2,1}(\bar{\Omega} \times(0, \infty])$; moreover, $u, v, w$ are nonnegative on $\bar{\Omega} \times(0, \infty)$ and $\|u(\cdot, t)\|_{L^{\infty}}+\|v(\cdot, t)\|_{L^{\infty}}+\|w(\cdot, t)\|_{L^{\infty}}$ are uniformly bounded for $t \in(0, \infty)$.

Our result in Theorem 1.2 extends the works of [22, 27] and [26]by establishing the global existence and boundedness of positive classical solutions to the fully parabolic system (1.1) over 2D without assuming the smallness of chemotactic rates or initial cellular populations. Therefore, it supports the study of spatial-temporal behaviors of positive solutions to (1.1), such as the formation of stable or time-periodic spatially-inhomogeneous patterns, at least when the domain is two-dimensional. See our numerical simulations in Section 5. Our results indicate that blow-up is inhibited by the logistic growth over two dimensional bounded domains. However it is worthwhile to point out that logistic growth is insufficient to prevent blow-ups in higher space dimension for chemotaxis model. See [30].

Without loss of our generality, we shall assume that $\tau=1$ in this paper, and Theorem 1.2 and our arguments hold for $\tau=0$. In the sequel, $C$ and $C_{i}$ denote time-independent positive constants that may vary between lines.

\section{Local existence and preliminary results}

In this section, we study the local existence of classical solutions to (1.1) and collect some basic facts for later use. First of all, we apply the well-known results of Amann [2, 3] to obtain the local existence of (1.1) which states as follows.

Theorem 2.1. Let $\tau=1$ and assume that for $i=1,2, a_{i}, d_{i}, \mu_{i}>0$ and $\chi_{i} \in \mathbb{R}$. Suppose that $u_{0} \in C^{\alpha}, v_{0} \in C^{\alpha}$ and $w_{0} \in W^{2, p}$ for some $\alpha \in(0,1)$ and $p>2$ satisfying $u_{0}, v_{0}, w_{0} \geq$ $0, \not \equiv 0$ in $\Omega$. Then there exist $T_{\max } \in(0, \infty]$ and a unique triple of nonnegative functions $(u(x, t), v(x, t), w(x, t)) \in C^{0}\left(\bar{\Omega} \times\left[0, T_{\max }\right]\right) \cap C^{2,1}\left(\bar{\Omega} \times\left(0, T_{\max }\right]\right)$ which solve (1.1) classically in $\Omega \times\left(0, T_{\max }\right)$. Moreover, there exists a positive constant such that

$$
\|u(\cdot, t)\|_{L^{1}(\Omega)}+\|v(\cdot, t)\|_{L^{1}(\Omega)}+\|w(\cdot, t)\|_{L^{1}(\Omega)} \leq C, \forall t \in\left(0, T_{\max }\right) .
$$

Furthermore, if $\sup _{s \in(0, t)}\|u(\cdot, s)\|_{L^{\infty}}+\|v(\cdot, s)\|_{L^{\infty}}+\|w(\cdot, s)\|_{L^{\infty}}$ is bounded for $t \in\left(0, T_{\max }\right)$, then $T_{\max }=\infty$ and $(u, v, w)$ is global.

Proof. System (1.1) can be rewritten into the abstract form as follows:

$$
\left(\begin{array}{c}
u \\
v \\
w
\end{array}\right)_{t}=\nabla \cdot\left[\mathcal{A} \nabla\left(\begin{array}{c}
u \\
v \\
w
\end{array}\right)\right]+\left(\begin{array}{c}
\left(a_{1}-b_{u}-c_{1} v\right) u \\
\left(a_{2}-b_{2} u-c_{2} v\right) v \\
-\lambda w+u+v
\end{array}\right),
$$

where

$$
\mathcal{A}=\left(\begin{array}{ccc}
d_{1} & 0 & -\chi_{1} u \\
0 & d_{2} & -\chi_{2} v \\
0 & 0 & 1
\end{array}\right)
$$

Since all the eigenvalues of $\mathcal{A}$ are positive, system (2.2) is normally parabolic and it is a triangular system, then (i) follows from Theorem 7.3 and Theorem 9.3 of [2] and (ii) follows from Theorem 5.2 in [3]. The regularities in this Theorem are immediate consequences of the standard parabolic estimates. Finally the nonnegativity of $(u, v, w)$ follows from parabolic Maximum Principles-see [14]. 
Next we have the following lemma due to the well-known smoothing properties of the operator $-\Delta+1$ and embeddings between the analytic semigroups generated by $\left\{e^{t \Delta}\right\}_{t \geq 0}$. We refer the reader to $[11,13,28,29]$ for references.

Lemma 2.2. Suppose that $\Omega \subset \mathbb{R}^{2}$ is a bounded domain with smooth boundary $\partial \Omega$. Let $(u, v, w)$ be the solution of (1.1) over $\left(0, T_{\max }\right)$. For $1 \leq p, q \leq \infty$, there exists a constants $C$ dependent on $\left\|w_{0}\right\|_{L^{q}(\Omega)}$ and $|\Omega|$ such that

$$
\|w(\cdot, t)\|_{W^{1, q}} \leq C\left(1+\sup _{s \in(0, t)}\left(\|u(\cdot, t)\|_{L^{p}}+\|v(\cdot, t)\|_{L^{p}}+\|w(\cdot, t)\|_{L^{p}}\right)\right), \forall t \in\left(0, T_{\max }\right),
$$

where $q \in\left[1, \frac{2 p}{2-p}\right)$ if $p \in[1,2), q \in(1, \infty)$ if $p=2$ and $q=\infty$ if $p>2$.

Proof. We write $w$-equation into the following abstract form

$$
w(\cdot, t)=e^{(\Delta-1) t} w_{0}+\int_{0}^{t} e^{(\Delta-1)(t-s)}((1-\lambda) w(\cdot, s)+u(\cdot, s)+v(\cdot, s)) d s .
$$

Applying Lemma 1.3 in [28] on (2.4), we have that such that for $1 \leq p, q \leq \infty$, there exists a positive constant $C$ such that

$$
\|w(\cdot, t)\|_{W^{1, q}} \leq C\left(1+\int_{0}^{t} e^{-\nu(t-s)}(t-s)^{-\frac{1}{2}-\frac{N}{2}\left(\frac{1}{p}-\frac{1}{q}\right)}\|(1-\lambda) w(\cdot, s)+u(\cdot, s)+v(\cdot, s)\|_{L^{p}} d s\right),
$$

where $\nu$ is the first Neumann eigenvalue of $-\Delta$. On the other hand, we see that

$$
\sup _{t \in(0, \infty)} \int_{0}^{t} e^{-\nu(t-s)}(t-s)^{-\frac{1}{2}-\frac{N}{2}\left(\frac{1}{p}-\frac{1}{q}\right)} d s<\infty, \text { if } q \in\left(1, \frac{N p}{N-p}\right),
$$

therefore (2.3) follows from (2.5).

The following result is an immediate consequence of (2.1) and (2.3).

Corollary 1. Under the same conditions in Lemma 2.2, there exists a positive constant $C$ such that for all $q \in[1,2)$

$$
\|w(\cdot, t)\|_{W^{1, q}} \leq C, \forall t \in\left(0, T_{\max }\right) .
$$

According to Lemma 2.2, we see that the boundedness of $\|(u, v)\|_{L^{p}}$ for some $p>2$ leads us to the boundedness of $\|\nabla w\|_{L^{\infty}}$ hence that of $\|w\|_{L^{\infty}}$. There are two well-established methods in proving the $L^{p}$-boundedness of the cellular population densities in literature. The first method is to construct time-monotone Lyapunov-functional of system (1.1), which also provides with some asymptotic properties of the solutions. The other is to use iterations on $L^{p}$-estimates of the solutions. Very recently, it is rigorously proved in [27] that the one-dimensional (1.1) has timeperiodic solutions when there are cellular growths, and time-monotone Lyapunov functional only exists for (1.1) in the absence of cellular growth, i.e., when $\mu_{1}=\mu_{2}$. Our numerical simulations also demonstrate that system (1.1) admits time-periodic spatially inhomogeneous solutions, which indicates the lack of time-monotone Lyapunov-functional. Our proof of the boundedness relies on the combined applications of estimates on the energy-type functional and the standard $L^{p}$-iterations.

\section{A-prior estimates}

According to Theorem 2.1, in order to prove the global existence of $(u, v, w)$ over $\Omega \times(0, \infty)$, we only need to establish the uniform boundedness of $\|u\|_{L^{\infty}}+\|v\|_{L^{\infty}}+\|w\|_{L^{\infty}}$. Indeed, we shall 
see that it is sufficient to find a bound on $\|\nabla w\|_{L^{\infty}}$ hence that of $\|u\|_{L^{p}}+\|v\|_{L^{p}}+\|w\|_{L^{p}}$ for some $p \geq 2$ thanks to Lemma 2.2. First of all, we introduce several entropy-type inequalities to estimate weighted functions $\|u \ln u\|_{L^{1}},\|v \ln v\|_{L^{1}}$ and $\|\nabla w\|_{L^{2}}$, etc. Our estimates rely on Gagliardo-Nirenberg interpolation inequality for which [11, 13] are good references.

Lemma 3.1. Let $\Omega \subset \mathbb{R}^{2}$ be a bounded domain with smooth boundary $\partial \Omega$. Assume that $\tau=1$, $a_{i}, d_{i}, \mu_{i}>0$ and $\chi_{i} \in \mathbb{R}$ for $i=1,2$. Suppose that the initial data satisfied the conditions in Theorem 2.1 and $(u, v, w)$ are the local solutions to (1.1) over $\Omega \times\left(0, T_{\max }\right)$. Then there exists a positive constant $C$ independent of $T_{\max }$ such that

$$
\|u \ln u\|_{L^{1}(\Omega)}+\|v \ln v\|_{L^{1}(\Omega)}+\|\nabla w\|_{L^{2}(\Omega)}<C, \forall t \in\left(0, T_{\max }\right) .
$$

Proof. In light of $u$-equation in (1.1), we have from the integration by parts and Young's inequality that

$$
\begin{aligned}
\frac{d}{d t} \int_{\Omega} u \ln u & =\int_{\Omega}(\ln u+1) u_{t} \\
& =\int_{\Omega}\left(\nabla \cdot\left(d_{1} \nabla u-\chi_{1} u \nabla w\right)\right)(\ln u+1)+\mu_{1} \int_{\Omega}\left(1-u-a_{1} v\right) u(\ln u+1) \\
& \leq-d_{1} \int_{\Omega} \frac{|\nabla u|^{2}}{u}+\chi_{1} \int_{\Omega} \nabla u \cdot \nabla w-\frac{\mu_{1}}{2} \int_{\Omega} u^{2} \ln u+C_{1}, \\
& \leq-\frac{d_{1}}{2} \int_{\Omega} \frac{|\nabla u|^{2}}{u}-\frac{\mu_{1}}{4} \int_{\Omega} u^{2} \ln u+\int_{\Omega}|\nabla w|^{4}+C_{2},
\end{aligned}
$$

where $C_{1}$ and $C_{2}$ are positive constants. Applying the same arguments on $v$-equation gives rise to

$$
\frac{d}{d t} \int_{\Omega} v \ln v \leq-\frac{d_{2}}{2} \int_{\Omega} \frac{|\nabla v|^{2}}{v}-\frac{\mu_{2}}{4} \int_{\Omega} v^{2} \ln v+\int_{\Omega}|\nabla w|^{4}+C_{3} .
$$

where $C_{3}$ is a positive constant. Moreover, we have from straightforward calculations that

$$
\begin{aligned}
\frac{1}{2} \frac{d}{d t} \int_{\Omega}|\nabla w|^{2} & =\int_{\Omega} \nabla w \cdot \nabla w_{t}=-\int_{\Omega} \Delta w w_{t}=-\int_{\Omega} \Delta w(\Delta w-\lambda w+u+v) \\
& =-\int_{\Omega}|\Delta w|^{2}-\int_{\Omega} \Delta w(-\lambda w+u+v) \\
& \leq-\frac{1}{4} \int_{\Omega}|\Delta w|^{2}+\lambda^{2} \int_{\Omega} w^{2}+\int_{\Omega} u^{2}+\int_{\Omega} v^{2} .
\end{aligned}
$$

On the other hand, in light of Corollary 1, we have from Gagliardo-Nirenberg interpolation and Hölder's inequalities that for any $\epsilon>0$, there exists $C(\epsilon)>0$ such that

$$
\|\nabla w\|_{L^{4}}^{4} \leq \epsilon\|\Delta w\|_{L^{2}}^{2}+C(\epsilon) .
$$

Adding up (3.2)-(3.4), we conclude from (3.5) that

$$
\frac{d}{d t}\left(\int_{\Omega} u \ln u+\int_{\Omega} v \ln v+\frac{1}{2} \int_{\Omega}|\nabla w|^{2}\right)+\frac{d_{1}}{4} \int_{\Omega} \frac{|\nabla u|^{2}}{u}+\frac{d_{2}}{4} \int_{\Omega} \frac{|\nabla v|^{2}}{v}+\frac{1}{8} \int_{\Omega}|\Delta w|^{2} \leq C_{4},
$$

where we have applied the fact from algebra that $-\frac{\mu_{1}}{4} u^{2} \ln u+u$ and $-\frac{\mu_{2}}{4} v^{2} \ln v+v$ are bounded from above by a uniform constant. Therefore (3.6) implies (3.1) through Gronwall's lemma. This completes the proof the lemma.

Lemma 3.2. Under the same conditions in Lemma 3.1, there exists a constant $C>0$ such that

$$
\|u(\cdot, t)\|_{L^{2}}+\|v(\cdot, t)\|_{L^{2}} \leq C, \forall t \in\left(0, T_{\max }\right) .
$$


Proof. We have from the integration by parts that

$$
\begin{aligned}
\frac{1}{2} \frac{d}{d t} \int_{\Omega} u^{2} & =-d_{1} \int_{\Omega}|\nabla u|^{2}-\frac{\chi_{1}}{2} \int_{\Omega} u^{2} \Delta w+\mu_{1} \int_{\Omega}\left(1-u-a_{1} v\right) u^{2} \\
& \leq-d_{1} \int_{\Omega}|\nabla u|^{2}+\frac{\chi_{1}}{2}\|u\|_{L^{3}}^{2}\|\Delta w\|_{L^{3}}+\mu_{1} \int_{\Omega}\left(1-u-a_{1} v\right) u^{2} .
\end{aligned}
$$

To estimate (3.8), we apply Lemma 3.5 of [18] with $p=3$ to have that for any $\epsilon>0$, there exists a positive constant $C(\epsilon)$ such that $\|u\|_{L^{3}} \leq \epsilon\|\nabla u\|_{L^{2}}^{\frac{2}{3}}\|u \ln u\|_{L^{1}}^{\frac{1}{3}}+C(\epsilon)\left(\|u \ln u\|_{L^{1}}+\|u\|_{L^{1}}^{\frac{1}{3}}\right)$. This fact and (3.1) imply that

$$
\|u\|_{L^{3}}^{2} \leq\left(\epsilon\|\nabla u\|_{L^{2}}^{2}+C(\epsilon)\right)^{\frac{2}{3}} .
$$

Thanks to the Gagliardo-Nirenberg inequality and (3.1), we have that

$$
\|\Delta w\|_{L^{3}} \leq C(\epsilon)\left(\|\nabla \Delta w\|_{L^{2}}^{\frac{2}{3}}\|\nabla w\|_{L^{2}}^{\frac{1}{3}}+\|\nabla w\|_{L^{2}}\right) \leq C(\epsilon)\left(\|\nabla \Delta w\|_{L^{2}}^{\frac{2}{3}}+1\right) .
$$

Combining (3.9) and (3.10), we infer from Young's inequality that

$$
\|u\|_{L^{3}}^{2}\|\Delta w\|_{L^{3}} \leq \frac{2 \epsilon}{3}\|\nabla u\|_{L^{2}}^{2}+\frac{\epsilon}{3}\|\nabla \Delta w\|_{L^{2}}^{2}+C_{5},
$$

therefore (3.8) give rises to

$$
\frac{1}{2} \frac{d}{d t} \int_{\Omega} u^{2}+\frac{d_{1}}{2} \int_{\Omega}|\nabla u|^{2} \leq \frac{\chi_{1} \epsilon}{6} \int_{\Omega}|\nabla \Delta w|^{2}+\mu_{1} \int_{\Omega}\left(1-u-a_{1} v\right) u^{2}+C_{6},
$$

where $\epsilon$ is taken to be smaller than $\frac{3 d_{1}}{2 \chi_{1}}$. By the same arguments, we can show that

$$
\frac{1}{2} \frac{d}{d t} \int_{\Omega} v^{2}+\frac{d_{2}}{2} \int_{\Omega}|\nabla v|^{2} \leq \frac{\chi_{2} \epsilon}{6} \int_{\Omega}|\nabla \Delta w|^{2}+\mu_{2} \int_{\Omega}\left(1-a_{2} u-v\right) v^{2}+C_{7} .
$$

On the other hand, we operate the $w$-equation in (1.1) by $\nabla$ and test the new equation by $-\nabla \Delta w$ over $\Omega$ by parts, then it follows from Young's inequality that

$$
\begin{aligned}
\frac{1}{2} \frac{d}{d t} \int_{\Omega}|\Delta w|^{2} & =\int_{\Omega} \Delta w \Delta w_{t}=-\int_{\Omega} \nabla \Delta w \cdot \nabla w_{t} \\
& =-\int_{\Omega}|\nabla \Delta w|^{2}-\int_{\Omega} \nabla \Delta w \nabla(\Delta w-\lambda w+u+v) \\
& \leq-\frac{1}{4} \int_{\Omega}|\nabla \Delta w|^{2}+\lambda^{2} \int_{\Omega}|\nabla w|^{2}+\int_{\Omega}|\nabla u|^{2}+\int_{\Omega}|\nabla v|^{2} .
\end{aligned}
$$

Multiplying (3.11) by $\frac{2}{d_{1}},(3.12)$ by $\frac{2}{d_{2}}$ and then adding them to (3.13), we conclude that

$$
\frac{d}{d t}\left(\frac{1}{d_{1}} \int_{\Omega} u^{2}+\frac{1}{d_{2}} \int_{\Omega} v^{2}+\frac{1}{2} \int_{\Omega}|\Delta w|^{2}\right)+\frac{1}{8} \int_{\Omega}|\nabla \Delta w|^{2}+\int_{\Omega} u^{2}+\int_{\Omega} v^{2} \leq C .
$$

Therefore (3.7) follows from (3.14) thanks to Gronwall's inequality.

\section{Existence of global-in-time solutions}

We now prove Theorem 1.2 which establishes the existence and boundedness of classical solutions to $(1.1)$ over $2 \mathrm{D}$. 
Proof of Theorem 1.2. To prove Theorem 1.2, we want to show that $\|(u(\cdot, t), v(\cdot, t))\|_{L^{\infty}}$ is uniformly bounded for all $t \in\left(0, T_{\max }\right)$, then the boundedness of $\|w(\cdot, t)\|_{L^{\infty}}$ follows from (2.3) taking $p$ large. Moreover, according to Theorem 2.1, we have that $T_{\max }=\infty$ and the existence part of Theorem 1.2 follows. Moreover, one can apply the standard parabolic boundary $L^{p}$-estimates and Schauder estimates in $[9,14]$ to show that $u_{t}, v_{t}, w_{t}$ and all spatial partial derivatives of $u, v$ and $w$ up to order two are bounded on $\bar{\Omega} \times[0, \infty)$, therefore $(u, v, w)$ have the regularities stated in Theorem 1.2.

Taking $p=2$ in Lemma 2.2 and in light of (3.7), we see that for any $q \in[2, \infty),\|\nabla w(., t)\|_{L^{q}}$ is uniformly bounded for all $t \in\left(0, T_{\max }\right)$. For each $p \geq 2$, we assume that $\|\nabla w(., t)\|_{L^{2(p+1)}} \leq 1$ without loss of our generality. Then we have from straightforward calculations that

$$
\frac{1}{p} \frac{d}{d t} \int_{\Omega} u^{p}=-\frac{4 d_{1}(p-1)}{p^{2}} \int_{\Omega}\left|\nabla u^{\frac{p}{2}}\right|^{2}+\frac{2 \chi_{1}(p-1)}{p} \int_{\Omega} u^{\frac{p}{2}} \nabla u^{\frac{p}{2}} \cdot \nabla w+\mu_{1} \int_{\Omega}\left(1-u-a_{1} v\right) u^{p} .
$$

To estimate (4.1), we first have from Young's inequality that

$$
u^{\frac{p}{2}} \nabla u^{\frac{p}{2}} \cdot \nabla w \leq \epsilon\left|\nabla u^{\frac{p}{2}}\right|^{2}+\epsilon\left(u^{\frac{p}{2}}\right)^{\frac{2(p+1)}{p}}+C(\epsilon)|\nabla w|^{2(p+1)},
$$

where $\epsilon>0$ is a constant to be determined and $C(\epsilon)>0$ is also a constant. Moreover, since $v>0$ we have that there exists $C_{0}>0$ such that $\mu_{1}\left(1-u-a_{1} v\right) u^{p}<-\frac{\mu_{1}}{2} u^{p+1}+C_{0}$. Now (4.1) implies that

$$
\begin{aligned}
\frac{1}{p} \frac{d}{d t} \int_{\Omega} u^{p} & \leq-\frac{4 d_{1}(p-1)}{p^{2}} \int_{\Omega}\left|\nabla u^{\frac{p}{2}}\right|^{2}+\frac{2 \chi_{1}(p-1)}{p} \int_{\Omega} u^{\frac{p}{2}} \nabla u^{\frac{p}{2}} \cdot \nabla w-\frac{\mu_{1}}{2} \int_{\Omega} u^{p+1}+\mu_{1} C_{0}|\Omega| \\
& \leq-\frac{4 d_{1}(p-1)}{p^{2}} \int_{\Omega}\left|\nabla u^{\frac{p}{2}}\right|^{2}+\frac{2 \chi_{1}(p-1)}{p} \int_{\Omega}\left(\epsilon u^{p+1}+\epsilon\left|\nabla u^{\frac{p}{2}}\right|^{2}\right)-\frac{\mu_{1}}{2} \int_{\Omega} u^{p+1}+C(\epsilon) \\
& \leq\left(-\frac{4 d_{1}(p-1)}{p^{2}}+\frac{2 \chi_{1}(p-1) \epsilon}{p}\right) \int_{\Omega}\left|\nabla u^{\frac{p}{2}}\right|^{2}+\left(\frac{2 \chi_{1}(p-1) \epsilon}{p}-\frac{\mu_{1}}{2}\right) \int_{\Omega} u^{p+1}+C(\epsilon) \\
& \leq-\frac{2 d_{1}(p-1)}{p^{2}} \int_{\Omega}\left|\nabla u^{\frac{p}{2}}\right|^{2}-\frac{\mu_{1}}{4} \int_{\Omega} u^{p+1}+C(\epsilon),
\end{aligned}
$$

where $\epsilon$ is chosen to be a fixed but sufficiently small positive constant. After applying on (4.2) the standard Moser-Alikakos $L^{p}$-iteration due to [1] (see Lemma A1 in [21] e.g.), we can show that there exists a constant $C>0$ such that $\|u(\cdot, t)\|_{L^{\infty}} \leq C, \forall t \in\left(0, T_{\max }\right)$. Similarly, we can prove the uniform boundedness of $\|v(\cdot, t)\|_{L^{\infty}}$ by exact the same calculations. The completes the proof of Theorem 1.2.

Remark 1. As is well known, the $L^{p}$-boundedness of $u$ and $v$, for some $p>N$, in Lemma 3.2 is sufficiently to show the boundedness of their $L^{\infty}$-norms for general Keller-Segel system. However, the logistic decay in (1.1) is necessary to prove Lemma 3.2 hence our main result. Moreover, finite blow-up is possible if there is no cellular death, in particular when the initial population is large, even over two-dimensional domains, bounded or unbounded. According to Lemma 2.2, we know that $\|\nabla w(., t)\|_{L^{q}}$ is bounded for any $q \in[1, \infty)$. Then we can apply (4.2) to show the boundedness of $\|u(., t)\|_{L^{p}}$ for $p=4$ for example. Then we can conclude from (2.3) that $\|\nabla w(., t)\|_{L^{\infty}}$ is bounded and then the proof carries over for any $p=2^{k}, k=2,3, \ldots$ recursively.

\section{Numerical studies and Discussions}

In this section, we present numerical simulations to illustrate the spatial-temporal behaviors of positive solutions to system (1.1) over 2D. Our numerics support the theoretical results and demonstrate that system (1.1) has very rich dynamics. We mainly investigate in this part 
the formation of nontrivial patterns in (1.1) such as spikes, layers, etc. In particular, we are concerned about the effect of system parameters such as chemotaxis rate, domain size, etc on the pattern formation. These concentrating patterns can be used to simulate the cellular aggregation phenomenon in chemotaxis. To manifest our results, we choose $\Omega$ to be $2 \mathrm{D}$ square and choose different sets of system parameters. In all these examples, we take $a_{1}=a_{2}=0.5$ and the initial data to be small perturbations from $(\bar{u}, \bar{v}, \bar{w})=\left(\frac{2}{3}, \frac{2}{3}, \frac{4}{3}\right)$. We present only the formations of $u(x, y, t)$ while similar spatial-temporal behaviors of $v$ and $w$ are observed in our experiments.

In Figure 1, we choose in (1.1) $d_{1}=d_{2}=d_{3}=\lambda=1, \chi_{1}=\chi_{2}=5$ and take the initial conditions to be $\left(u_{0}, v_{0}, w_{0}\right)=(\bar{u}, \bar{v}, \bar{w})+(0.001,0.001,0.001) \cos 2 x \cos 2 y$. Graphes of $u(x, y, t)$ at time $t=0,2,5,8,10,20,30$ are plotted and we can easily observe the formation of aggregated patterns from spatially homogeneously distributed solution, which eventually develop into a stable patterns with multiple interior spikes. Our simulations in Figure 1 demonstrate the global existence and boundedness of solutions to (1.1); moreover, they suggest that the spatially homogeneous solution $(\bar{u}, \bar{v}, \bar{w})$ loses its stability to stable spatially inhomogeneous patterns.
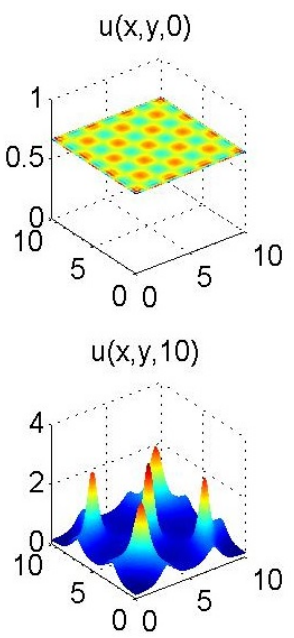

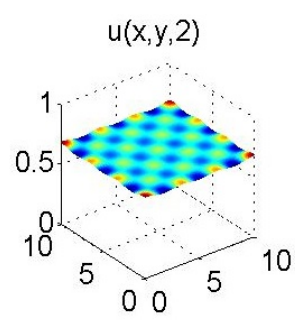

$u(x, y, 20)$

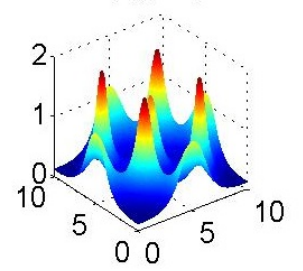

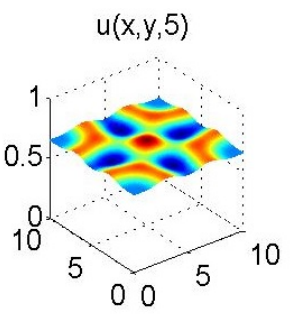

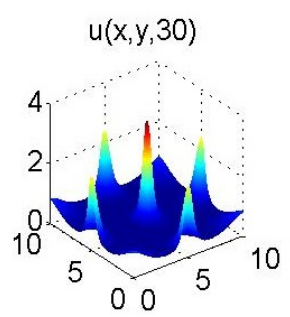

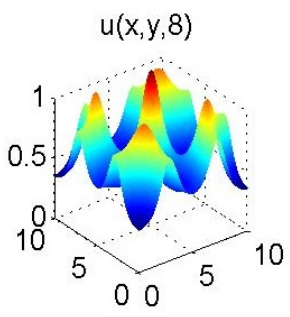

Stable $u(x, y)$

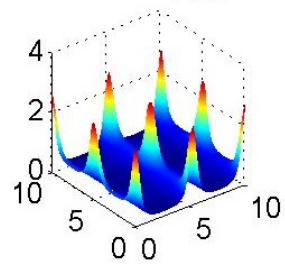

Figure 1: The evolution and formation of spiky patterns of $u(x, y, t)$, where the initial data are chosen to small perturbations from the homogeneous solution $(\bar{u}, \bar{v}, \bar{w})=\left(\frac{2}{3}, \frac{2}{3}, \frac{4}{3}\right)$.

In Figure 2, we present numerical simulations on the dynamics of spatially-inhomogeneous time-periodic patterns to system (1.1) with a period $T \approx 3.6$. We choose the system parameters to be $d_{1}=d_{2}=d_{3}=\lambda=1, \chi_{1}=\chi_{2}=20$, while the initial conditions are $(\bar{u}, \bar{v}, \bar{w})+$ $(0.001,0.001,0.001) \cos 2 x \cos 2 y$. Compared with Figure 1 , numerical experiments in Figure 2 suggest that system (1.1) also admits periodic patterns with properly chosen chemotaxis rates $\chi_{1}$ and $\chi_{2}$. The results indicate that $(\bar{u}, \bar{v}, \bar{w})$ loses its stability to spatially inhomogeneous stable patterns through Hopf bifurcation. However, similar as rigorous analysis for the pattern formation in Figure 1, it is beyond the scope of this paper to investigate the Hopf bifurcation analysis of (1.1). We want to point to point out that rigorous Hopf bifurcation analysis of (1.1) over $\Omega=(0, L)$ is performed in [27], as well as the stability of the time-periodic solutions.

Next we investigate the effect of domain size on the shape of stable patterns for (1.1). In particular, we choose $\Omega=(0, L) \times(0, L)$ with $L=5,10,15$ and 20 respectively. Systems parameters are selected to be $d_{1}=d_{2}=d_{3}=\lambda=5, \chi_{1}=\chi_{2}=10$, and initial data are taken to the same as in Figure 2. Our simulations suggest that large domains support stable patterns with more spikes than small domains. Indeed, if $u(x, y)$ is a solutions to (1.1) over $\Omega=(0, L) \times(0, L)$, then $u(2 L-x, 2 L-y)$ is a solution over $\Omega=(0,2 L) \times(0,2 L)$ thanks to the Neumann boundary conditions, therefore one can construct solutions over even larger domains 


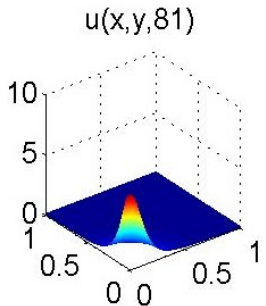

$u(x, y, 97.0)$

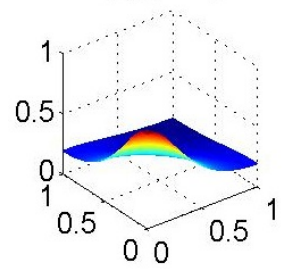

$u(x, y, 84.2)$

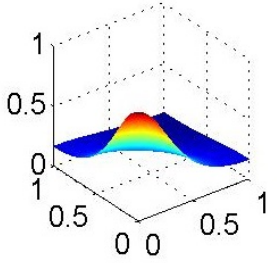

$u(x, y, 109.6)$

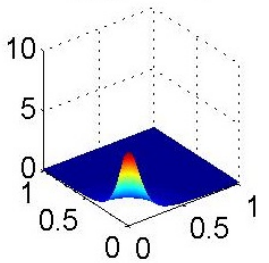

$u(x, y, 87.4)$

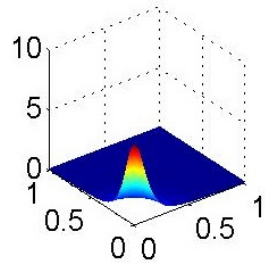

$u(x, y, 106.4)$

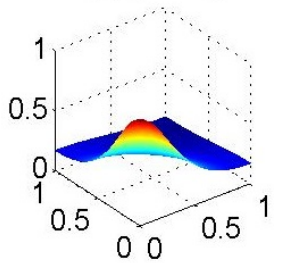

$u(x, y, 90.6)$

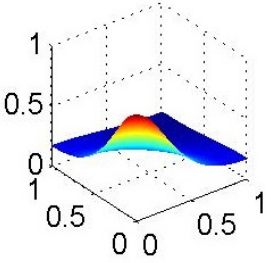

$u(x, y, 131.8)$

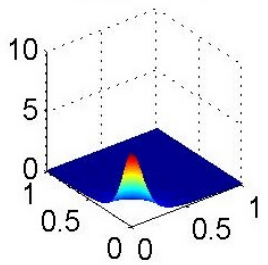

Figure 2: The evolution and formation of time-periodic spatially-inhomogeneous patterns to (1.1), where the system parameters and initial data are chosen to be the same as those for Figure 1 except for that $\chi_{1}=\chi_{2}=20$

by periodically reflecting and extending these solutions along domain boundaries.
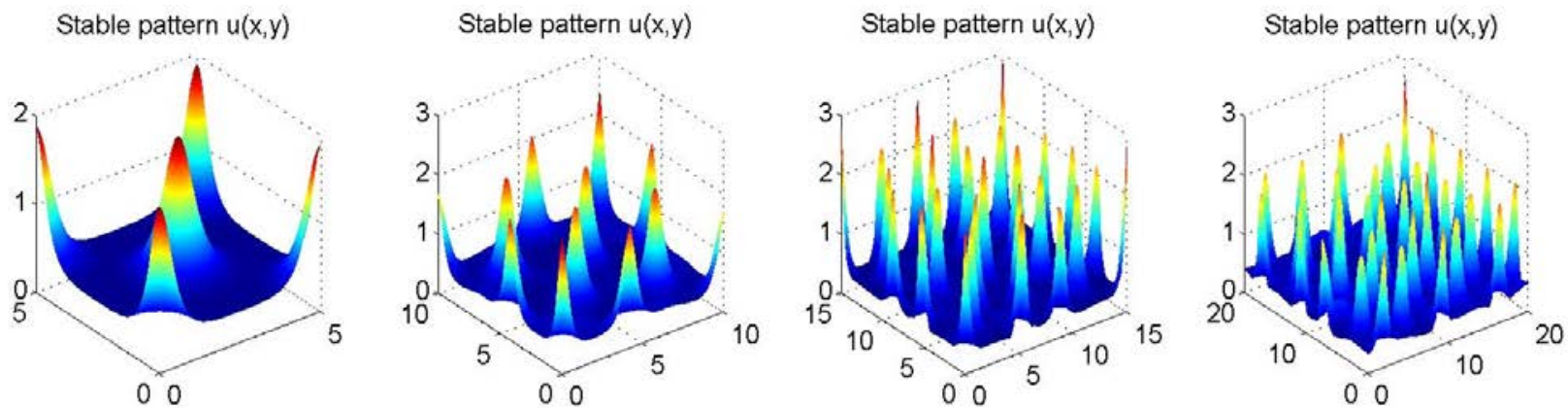

Figure 3: Stable patterns of $u(x, y)$ over 2D squares with side lengths $L=5,10,15$ and 20 respectively. Our simulations suggest that large domains support stable patterns with more aggregates than small domains.

Figure 4 presents numerical simulations to illustrate the effect of chemotaxis rate on the stable patterns. Parameters are $d_{1}=5, d_{2}=d_{3}=10, \lambda=1, \chi_{2}=100$ and the same initial data are chosen as in Figure 1. $\chi=50,100,150$ and 200 are chosen in each plot from the left to the right. Our numerics indicate that large chemotaxis rate support stable spikes with more aggregates; moreover, larger chemotaxis rate makes $u(x, y)$ concentrate at a higher amplitude.

Finally, we present in Figure 5 various interesting stable patterns $u(x, y)$ subject to the variations of initial data over $\Omega=(0,10) \times(0,10)$. The initial data are taken to be radially symmetric with a center $(0,0)$ in the first plot, symmetric in both $x$ and $y$ in the second plot, a periodic function of $x$ in the third plot, and radially symmetric with a center $(5,5)$ in the last plot. Then we observe that the same stable patterns are developed.

In this paper, we prove the global existence and boundedness of (1.1) over $\Omega \subset \mathbb{R}^{2}$ without assuming (1.2). Our work extends and complements the results in $[4,7,8,10]$ and $[22,26,27]$ in the reference. The result provides a complete understanding on the global existence of (1.1) 

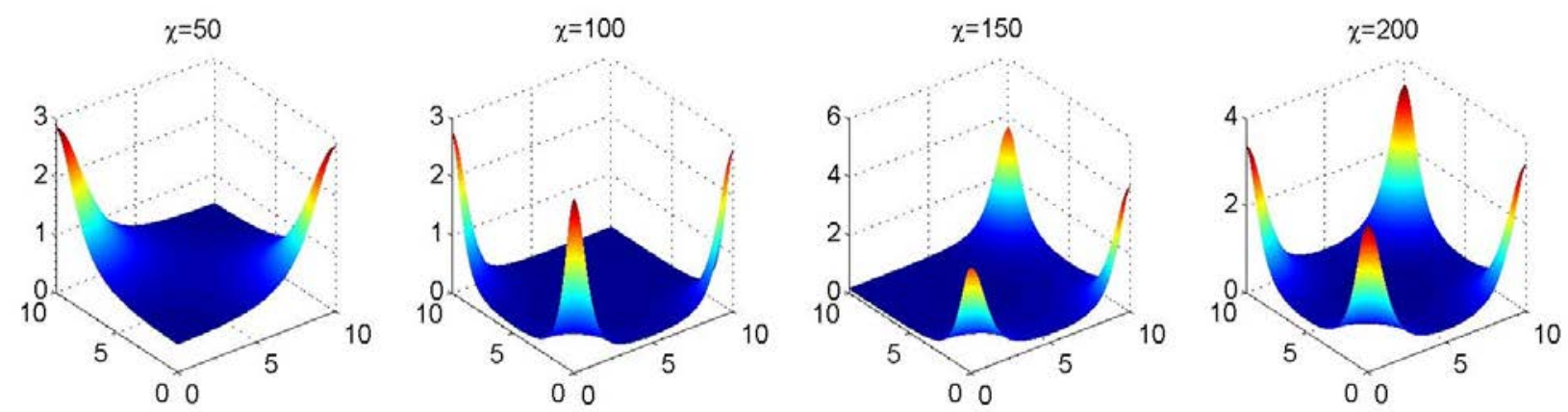

Figure 4: Stable pattern $u(x, y)$ for different chemotaxis rate $\chi_{1}$.
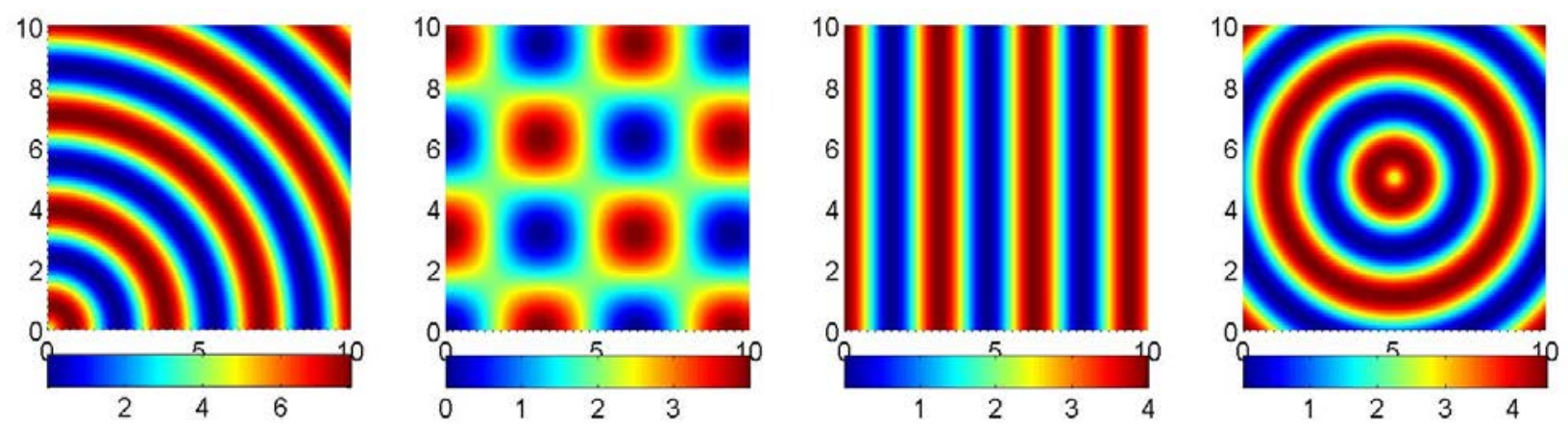

Figure 5: Various interesting stable patterns of $u(x, y)$ with different initial data.

in $2 \mathrm{D}$, and makes it possible to study the spatial-temporal behaviors and pattern formation in (1.1).

Our global existence result is heavily involved with the space dimension $N=2$ since $q$ can not taken to be arbitrarily large in (2.3) if $N \geq 2$. In higher space-dimensions, the Laplace operator may be insufficient to prevent finite or infinite-time blow-up of (1.1), in particular when the chemotaxis rates $\chi_{1}$ and $\chi_{2}$ are large. Therefore, it is interesting to probe the global existence of (1.1) over high-dimensional spaces, at least when $N=3$ which is biologically realistic.

We present various numerical simulations to demonstrate the existence of stable patterns and time-periodic spatially-inhomogeneous patterns over 2D. Our numerics suggest that the homogeneous steady state loses its stability to spatially inhomogeneous patterns either through steady state bifurcation or Hopf bifurcation. It is interesting to investigate the formation of these patterns through rigorous mathematical analysis, compared with the steady state bifurcation analysis in [26] and Hopf bifurcation analysis in [27]. Moreover, our experiments include the formation of various interesting stable patterns due to the effect of chemotaxis rate, domain size, or initial data. We postpone it for further studies on the mechanism of the formation of such patterns.

\section{Acknowledgement}

We would like to thank Prof. Qi Wang at SWUFE for pointing out this problem to us, as well as many stimulating discussions over the numerical simulations. 


\section{References}

[1] N. D. Alikakos, $L^{p}$ bounds of solutions of reaction-diffusion equations, Comm. Partial Differential Equations, 4 (1979), 827-868.

[2] H. Amann, Dynamic theory of quasilinear parabolic equations. II. Reaction-diffusion systems, Differential Integral Equations, 3 (1990), 13-75.

[3] H. Amann, Nonhomogeneous linear and quasilinear elliptic and parabolic boundary value problems, Function Spaces, differential operators and nonlinear Analysis, Teubner, Stuttgart, Leipzig, 133 (1993), 9-126.

[4] P. Biler, E. Espejo and I. Guerra, Blowup in higher dimensional two species chemotactic systems, Commun. Pure Appl. Anal, 12 (2013), 89-98.

[5] A. Chertock, A. Kurganov, X. Wang and Y. Wu, On a chemotaxis model with saturated chemotactic flux, Kinetic and Related Model, 5 (2012), 51-95.

[6] S. Childress and J. K. Percus, Nonlinear aspects of chemotaxis, Math. Bioscience, 56, (1981), 217-237.

[7] C. Conca, E. Espejo and K. Vilches, Remarks on the blowup and global existence for a two species chemotactic Keller-Segel system in $\mathbb{R}^{2}$, European J. Appl. Math, 22 (2011), 553-580.

[8] C. Conca, E. Espejo and K. Vilches, Sharp Condition for blow-up and global existence in a two species chemotactic Keller-Segel system in $\mathbb{R}^{2}$, European J. Appl. Math, 24 (2013), $297-313$.

[9] L.C. Evans "Partial Differential Equations, second edition", American Mathematical Society, (2010).

[10] E. Espejo, Angela Stevens and Juan J. L. Velázquez, Simultaneous finite time blow-up in a two-species model for chemotaxis, Analysis, 29 (2009), 317-338.

[11] D. Henry, "Geometric theory of semilinear parabolic equations", Springer-Verlag, BerlinNew York (1981).

[12] M. A. Herrero and J. J. L. Velazquez, Chemotactic collapse for the Keller-Segel model, Journal of Mathematical Biology, 35 (1996), 177-194.

[13] D. Horstmann and M. Winkler, Boundedness vs. blow-up in a chemotaxis system, J. Differential Equations, 215 (2005), 52-107.

[14] O.A. Ladyzenskaja, V.A. Solonnikov and N.N. Ural'ceva, "Linear and Quasi-Linear Equations of Parabolic Type", American Mathematical Society, (1968), 648 pages.

[15] Y. Lou W.-M. Ni, Diffusion, self-diffusion and cross-diffusion, J. Differential Equations, 131 (1996), 79-131.

[16] Y. Lou W.-M. Ni, Diffusion vs cross-diffusion: An elliptic approach, J. Differential Equations, 154 (1999), 157-190.

[17] V. Nanjundiah, Chemotaxis, signal relaying and aggregation morphology, Journal. Theor. Biol, 42 (1973), 63-105.

[18] T. Nagai, T. Senba and K. Yoshida, Application of the Trudinger-Moser inequality to a parabolic system of chemotaxis, Funkcial. Ekvac., 40 (1997), 411-433.

[19] W.-M. Ni and I. Takagi, On the shape of least enery solutions to a semilinear Neumann problem, Communication of Pure and Applied Math, 44 (1991), 819-851.

[20] W.-M. Ni and I. Takagi, Location of the peaks of least energy solutions to a semilinear Neumann problem, Duke Math Journal, 70 (1993), 247-281.

[21] Y. Tao and M. Winkler Boundedness in a quasilinear parabolicCparabolic KellerCSegel system with subcritical sensitivity, Journal of Differential Equations, 252 (2012), 692-715. 
[22] J.I. Tello and M. Winkler, Stabilization in a two-species chemotaxis system with a logistic source, Nonlinearity, 25 (2012), 1413-1425.

[23] Q. Wang, Global solutions of a Keller-Segel system with saturated logarithmic sensitivity function, Commun. Pure Appl. Anal., 14 (2015), 383-396.

[24] Q. Wang, Boundary spikes of a keller-segel chemotaxis system with saturated logarithmic sensitivity, Discrete Contin. Dyn. Syst.-Series B, 20 (2015), 1231-1250.

[25] Q. Wang, C. Gai and J. Yan, Qualitative analysis of a Lotka-Volterra competition system with advection, Discrete Contin. Dyn. Syst. 35 (2015), 1239-1284.

[26] Q. Wang, Lu Zhang, Jingyue Yang and Jia Hu, Global existence and steady states of a two competing species Keller-Segel chemotaxis model, Kinetic and Related Models, 8 (2015), 777-807.

[27] Q. Wang, Jingyue Yang and Lu Zhang, Time periodic and stable patterns of a two-competing-species Keller-Segel chemotaxis model: effect of cellular growth, preprint, http://arxiv.org/abs/1505.06463

[28] M. Winkler, Absence of collapse in a parabolic chemotaxis system with signal-dependent sensitivity, Math. Nachr, 283 (2010), 1664-1673.

[29] M. Winkler, Aggregation vs. global diffusive behavior in the higher-dimensional Keller-Segel model, Journal of Differential Equations, 248 (2010), 2889-2905.

[30] M. Winkler, Blow-up in a higher-dimensional chemotaxis system despite logistic growth restriction, J. Math. Anal. Appl., 384 (2011), 261-272. 